\title{
La mobilité domicile-travail des actifs de l'aire urbaine lyonnaise : une approche temporelle (1995-2015)
}

Home-To-Work Mobility of Lyon's Urban Area Workers: A Temporal Approach (1995-2015)

Nathalie Havet, Caroline Bayart et Patrick Bonnel

\section{(2) OpenEdition}

Journals

Édition électronique

URL : http://journals.openedition.org/travailemploi/9552

DOI : 10.4000/travailemploi.9552

ISSN : $1775-416 \mathrm{X}$

Éditeur

DARES - Ministère du Travail

Édition imprimée

Date de publication : 2 décembre 2019

Pagination : 47-74

ISSN : 0224-4365

Référence électronique

Nathalie Havet, Caroline Bayart et Patrick Bonnel, « La mobilité domicile-travail des actifs de l'aire urbaine lyonnaise : une approche temporelle (1995-2015) », Travail et Emploi [En ligne], 160 | 2019, mis en ligne le 01 novembre 2020, consulté le 25 mars 2021. URL : http://journals.openedition.org/ travailemploi/9552 ; DOI : https://doi.org/10.4000/travailemploi.9552 


\title{
La mobilité domicile-travail des actifs de l'aire urbaine lyonnaise : une approche temporelle (1995-2015)
}

\author{
Nathalie Havet, Caroline Bayart ${ }^{* *}$, Patrick Bonnel ${ }^{* * *}$
}

Ces dernières années, les grandes villes françaises ont connu un double phénomène de métropolisation et d'expansion spatiale. Dans ce contexte, la mobilité quotidienne des individus, et tout particulièrement celle liée au travail, constitue un enjeu central pour le développement des infrastructures. En s'appuyant sur les trois dernières Enquêtes ménages déplacements, cet article propose d'étudier l'évolution de la mobilité domicile-travail des actifs lyonnais depuis vingt ans (distance domicile-travail parcourue et usage de la voiture pour aller au travail). L'objectif est de déterminer si l'évolution des indicateurs de mobilité est davantage imputable à une modification de comportements des actifs ou à un changement structurel des caractéristiques de ces derniers, en intégrant une dimension temporelle dans les modèles économétriques. Si certaines différences de comportements de mobilité persistent, notamment selon le type d'emploi, l'utilisation de la voiture pour se rendre au travail a fortement évolué, mettant en évidence un effet de rattrapage chez les femmes et les professions et catégories socioprofessionnelles les moins élevées.

$\mathrm{D}$ urant les dernières décennies, le phénomène de métropolisation s'est accéléré en France. La métropolisation, ou « phase du développement urbain en rapport avec les dynamiques économiques contemporaines » (PASQUIER et al, 2011, p. 333), se caractérise par une concentration croissante de la population, de la production de richesses, des services stratégiques et des fonctions de commandement dans les agglomérations. L'agglomération de Lyon a été particulièrement concernée, puisque

\footnotetext{
* Université de Lyon, Université Claude Bernard Lyon 1, ISFA, Laboratoire SAF, Lyon, France ; nathalie.havet@ univ-lyon1.fr.

** Université de Lyon, Université Claude Bernard Lyon 1, ISFA, Laboratoire SAF, Lyon, France ; caroline.bayart@ univ-lyon1.fr.

*** Université de Lyon, CNRS, Laboratoire aménagement, économie, transports (LAET), ENTPE, Lyon, France ; patrick.bonnel@entpe.fr.
} 
le second pôle urbain de l'Hexagone a enregistré, entre 2011 et 2016, une croissance démographique annuelle moyenne de $1,1 \%$, alors qu'elle était de $0,4 \%$ au niveau de la France entière ${ }^{1}$. Une étude publiée par le Commissariat général à l'égalité des territoires (CGET) indique également que les zones d'emplois de Paris, Lyon, Bordeaux et Toulouse concentraient $22 \%$ de l'emploi total en France et $20 \%$ des embauches en 2015 (OBSERVATOIRE DES TERRITOIRES, 2016). Parallèlement à cette concentration, les villes ont connu une extension spatiale, encouragée par le développement des infrastructures à destination des transports collectifs et de la voiture ${ }^{2}$ et l'accroissement de la motorisation des individus ${ }^{3}$ (GEYIK, BONNEL, 2016). Ce développement s'est accompagné d'un étalement des activités liées au mode de vie urbain (zones d'activités économiques, infrastructures de transports, zones commerciales, parcs de loisirs, aéroports, etc.). En particulier, les lieux de résidence, de travail, de loisirs et de consommation se sont dissociés de manière croissante, ce qui a intensifié les déplacements entre territoires (CABRERA DElgado, Bonnel, 2016).

Dans ce contexte, la mobilité quotidienne des individus constitue un enjeu central, tant du point de vue socioéconomique (l'accès à l'emploi) qu'environnemental (la gestion durable des déplacements). Parmi les différents types de mobilité, la mobilité domicile-travail est celle qui a le plus bouleversé les territoires urbains des dernières décennies, puisque les lieux de résidence et d'exercice de l'activité professionnelle sont plus fréquemment disjoints aujourd'hui que par le passé. C'est pourquoi notre article se propose d'étudier l'évolution des déplacements quotidiens domicile-travail de la population en emploi, au sein d'une des plus grandes métropoles françaises, Lyon. Nous analysons des données désagrégées, collectées au niveau individuel (celui des travailleurs) et à l'échelle intra-urbaine. En effet, alors que la proportion d'actifs qui sortent de leur commune de résidence pour travailler, appelés navetteurs, est en croissance (64\% en 2013 contre $58 \%$ en 1999 au niveau national et $66 \%$ en 2013 contre $60 \%$ en 1999 pour la région Auvergne-Rhône-Alpes), habiter et travailler dans la même aire urbaine est encore, en France, le modèle dominant, puisque $78 \%$ des flux de navetteurs sont concentrés dans les 241 aires urbaines recensées par l'Insee (COUDÈnE, LEVY, 2016).

En nous appuyant sur les trois dernières vagues de l'Enquête ménages déplacements de Lyon (1995, 2006, 2015), nous étudions l'évolution des déplacements quotidiens domicile-travail des actifs qui résident et travaillent dans la métropole lyonnaise sur une période de vingt ans. Nous nous focalisons en particulier sur l'allongement des distances domicile-travail parcourues et sur le moindre usage de la voiture particulière, comme cela a été mis en évidence pour les jeunes adultes dans la littérature internationale (KuHNIMHOF et al., 2013 ; DARGAY et al., 2007), en lien avec la mise en place de politiques de transport urbain (OAKIL et al., 2016). Plus précisément, nous examinerons si les déterminants principaux de ces indicateurs de mobilité quotidienne

1. Poyet S. (2018), « La croissance démographique française tirée par les grandes villes », Le Figaro, 27 décembre.

2. Au moins au siècle dernier, car l'évolution de l'offre est plus contrastée depuis.

3. Au moins jusqu'à la fin du siècle dernier ; l'évolution est, là encore, plus contrastée au XXI siècle avec une poursuite de la motorisation au-delà de 40 ans mais une réduction chez les plus jeunes adultes. 
ont changé en vingt ans. L'idée est de dissocier, au sein des évolutions observées, ce qui pourrait être attribuable aux changements structurels des caractéristiques des actifs sur le périmètre considéré (évolution des professions et catégories socioprofessionnelles, des lieux de résidence, des situations familiales, etc.) de ce qui pourrait être imputable à des modifications de comportements. Pour tenter de saisir ces éventuels changements de comportements, nous estimons des modèles économétriques intégrant une dimension temporelle.

L'article s'organise comme suit. Dans un premier temps, nous présentons les données des Enquêtes ménages déplacements de Lyon et la construction de l'échantillon utilisé. Nous mettons en évidence dans un deuxième temps les évolutions qui ont eu lieu en vingt ans pour les distances domicile-travail et l'usage de la voiture par les actifs en emploi dans l'agglomération lyonnaise. Les différences entre changements structurels et modifications de comportements sont discutées dans un troisième temps.

\section{Les Enquêtes ménages déplacements de Lyon $(1995,2006,2015)$ : présentation de la méthodologie et de l'échantillon}

En France, les Enquêtes ménages déplacements $(E M D)$ représentent la principale source statistique pour analyser les pratiques de mobilité quotidienne en milieu urbain, leurs conséquences sociales et environnementales, et pour réfléchir au développement des infrastructures. Établies sur la base d'une méthodologie standardisée (CERTU, 2008) contrôlée par la direction technique Territoires et ville du Centre d'études et d'expertise sur les risques, l'environnement, la mobilité et l'aménagement (Cerema), ces enquêtes sont menées à grande échelle, de manière régulière (tous les dix ans environ) dans la plupart des grandes agglomérations françaises. L'objectif est notamment de disposer d'une bonne comparabilité des indicateurs de mobilité, dans le temps et entre les différentes agglomérations. Les personnes enquêtées sont interrogées sur l'ensemble de leurs déplacements réalisés la veille du jour de l'enquête, en semaine (lundi à vendredi). Les Centres d'étude technique de l'équipement (Cete) définissent le concept de déplacement par « le mouvement d'une personne, effectué pour un certain motif, sur une voie publique, entre une origine et une destination, selon une heure de départ et une heure d'arrivée, à l'aide d'un ou plusieurs moyens de transport »(CETE de Lyon, 2011, p. 14).

Pour l'étude menée ici, centrée sur l'évolution des déplacements domicile-travail dans l'aire urbaine lyonnaise, nous utilisons les trois vagues les plus récentes de l' $E M D$ de Lyon $(1995,2006,2015)$ en nous limitant à la population adulte, en emploi, âgée de 18 à $70 \mathrm{ans}^{4}$. Le périmètre des $E M D$ réalisées dans l'agglomération lyonnaise s'est

4. Les données pour l'EMD de Lyon sont traditionnellement collectées en face-à-face au domicile du ménage ou par téléphone. Le ménage constitue l'unité statistique d'échantillonnage de l'EMD : à l'issue de la réalisation d'une stratification géographique, un échantillon représentatif des ménages du périmètre d'investigation est enquêté. L'interrogation porte sur les déplacements de la veille du jour de l'enquête et ne concerne que les personnes de plus de 5 ans. 


\section{ENCADRÉ 1}

\section{Aire urbaine de Lyon et politiques de transport}

Le territoire de l'aire urbaine de Lyon a une structure monocentrique (BonNEL et al., 2003 ; BonNel et al., 2013). On distingue les communes du centre (Lyon et Villeurbanne), celles de la proche banlieue socialement assez différenciées (première couronne) et les communes périurbaines (deuxième couronne et troisième couronne est). Ces dernières s'étendent davantage à l'est qu'à l'ouest, où leur développement est restreint par la topographie (monts du Lyonnais). Si les habitants du centre et de la proche banlieue bénéficient d'un large accès au réseau de transports en commun, ceux des communes périurbaines restent en revanche fortement dépendants de la voiture particulière pour leurs déplacements quotidiens (GoYON et al., 2010).

La figure suivante présente les contours du Grand Lyon et l'évolution des périmètres des Enquêtes ménages déplacements (EMD) entre 1995 et 2015. Pour des raisons de comparabilité temporelle des résultats, seuls les comportements de mobilité des ménages résidant et travaillant dans le périmètre de l'enquête 1995 seront étudiés.

FIGURE - Découpage des périmètres des Enquêtes ménages déplacements $(1995,2006,2015)$

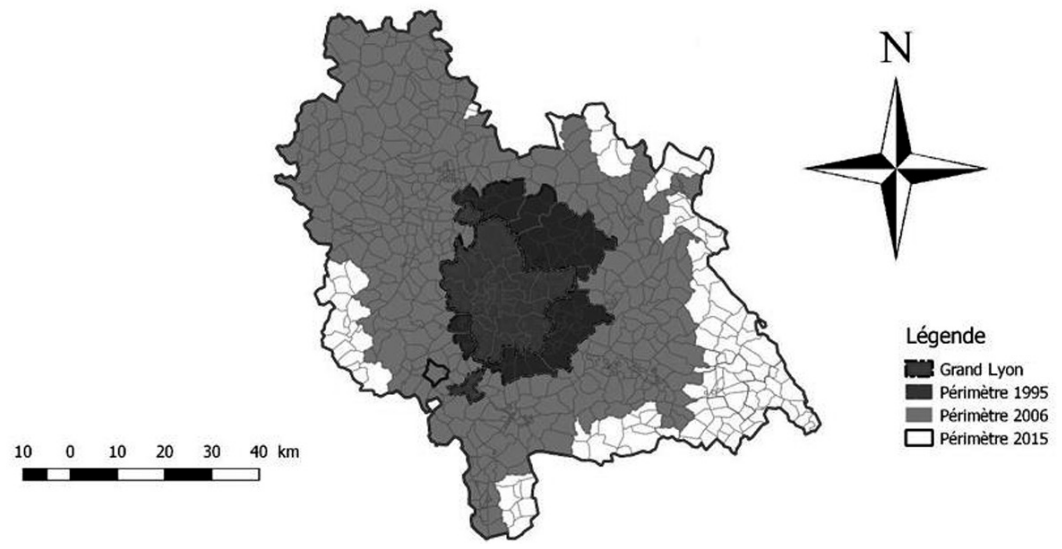

Le périmètre de l'EMD 1995 correspond approximativement au périmètre de l'autorité des transports publics urbains. Le réseau de transport en commun de l'agglomération lyonnaise est très dense et souvent considéré comme l'un des meilleurs réseaux français. Les infrastructures de transports collectifs se sont développées progressivement (inauguration du métro dans les années 1980, puis extension continue des lignes, création de six lignes de tramway, restructuration et hiérarchisation du réseau de bus avec le projet Atoubus en 2011) avec pour résultat une forte hausse des kilomètres parcourus. Plus précisément, sur la période 1995-2015, de nombreux investissements ont été réalisés sur l'agglomération. Deux des quatre lignes de métro ont été prolongées (vers l'ouest en 1997 et vers le sud en 2000 et 2013), deux lignes de tramway ont été mises en service en 2001, suivies de trois autres en 2006, 2009 et 2012. Une ligne de tram-train, le Rhône Express, dessert également l'aéroport depuis 2010. Concernant les modes doux, une offre de location de vélos en libre-service est proposée depuis 2005. 
progressivement élargi, dépassant l'aire urbaine de Lyon (encadré 1). Pour étudier l'évolution temporelle de la mobilité domicile-travail sans introduire de biais lié à une extension du territoire enquêté, toutes nos analyses sont menées à périmètre constant. Nous avons donc choisi le plus petit dénominateur commun, à savoir le périmètre de l'EMD 1995, qui correspond approximativement au périmètre de l'autorité des transports publics urbains. Ainsi, seuls les ménages résidant dans le périmètre de l'enquête 1995 sont comptabilisés dans les échantillons issus des enquêtes 2006 et 2015. En outre, nous devons ajouter une restriction supplémentaire et ne conserver pour l'analyse que les individus travaillant dans ce même périmètre (celui de 1995). Les localisations hors périmètre $E M D$ sont très peu renseignées et ne permettent pas de calculer la distance des déplacements domicile-travail avec un niveau de précision suffisant. Entre $6 \%$ et $15 \%$ des actifs selon les années sont ainsi exclus de nos analyses, et concernent plutôt les professions et catégories socioprofessionnelles (PCS) les plus élevées. Il faudra donc garder en tête cette spécificité lors des interprétations. Notre échantillon final est ainsi constitué de 16502 individus (5 411 pour 1995, 5763 pour 2006 et 5328 pour 2015), dont les caractéristiques (sociodémographiques, économiques, spatiales, et de motorisation) sont présentées dans le tableau 1.

Travailler à périmètre constant ne signifie pas que les caractéristiques socioéconomiques des personnes interrogées aux différentes éditions de l'EMD n'ont pas changé. Entre les enquêtes de 1995, 2006 et 2015, le tableau 1 met en évidence un vieillissement de la population, une diminution de la taille des ménages (augmentation des proportions de célibataires et de couples sans enfant, baisse de la part des familles avec enfant[s]) et une redensification du centre de l'agglomération. Après plusieurs décennies d'étalement urbain, nous assistons en effet à Lyon à une relocalisation des actifs vers le centre-ville (48\% des actifs en centre-ville en 2015 contre $32 \%$ en 1995, tableau 1), ce qui est une spécificité par rapport à d'autres grandes communes françaises comme Paris. La majorité des villes situées dans la couronne des grands pôles d'emplois enregistrent une croissance démographique plus forte que leur villecentre, illustrant le mouvement de périurbanisation (INSEE, 2019, pp. 216-18). À Lyon, ce mouvement a été contrebalancé par un processus de « recentrification », tendance notamment due à la politique de densification du centre, mais qui n'a pas concerné tous les ménages. Le choix de localisation résidentielle diffère en effet selon leurs caractéristiques socioéconomiques. Les jeunes adultes et actifs ayant un niveau d'études élevé et occupant un emploi qualifié (ce qui est souvent corrélé avec de hauts revenus) ont notamment davantage tendance à rejoindre le centre-ville, où ils peuvent accéder à une large offre d'emplois et d'activités de loisirs, accessibles par un réseau de transports en commun performant.

Plus généralement, sur cette période de vingt ans, et à l'image d' autres métropoles régionales comme Paris, Grenoble, Toulouse ou Montpellier, la structure des emplois de la métropole lyonnaise a connu des changements de fond, avec une diminution des emplois d'ouvriers et une concentration progressive d'emplois plus qualifiés. Le taux de chômage relativement faible de la métropole lyonnaise $(8,6 \%$ de la population 
TABLEAU 1 - Caractéristiques socioéconomiques des personnes interrogées lors des EMD de Lyon 1995, 2006 et 2015

\begin{tabular}{|c|c|c|c|}
\hline & 1995 & 2006 & 2015 \\
\hline \multicolumn{4}{|l|}{ Genre } \\
\hline Homme & 52,4 & 51,1 & 50,7 \\
\hline Femme & 47,6 & 48,9 & 49,3 \\
\hline Âge moyen, en années & 40,0 & 41,1 & 42,1 \\
\hline \multicolumn{4}{|l|}{ Composition du ménage } \\
\hline Célibataire & 13,6 & 15,0 & 19,6 \\
\hline Couple sans enfant & 20,3 & 20,8 & 24,8 \\
\hline Famille monoparentale & 7,0 & 9,5 & 9,0 \\
\hline Famille avec 1 enfant & 21,3 & 18,7 & 16,6 \\
\hline Famille avec 2 enfants & 24,4 & 22,4 & 19,5 \\
\hline Famille avec 3 enfants ou plus & 13,4 & 13,5 & 10,4 \\
\hline Présence d'enfant(s) de moins de 5 ans & 16,9 & 18,1 & 15,5 \\
\hline \multicolumn{4}{|l|}{ Situation de couple et statut du conjoint } \\
\hline Pas de conjoint & 20,5 & 24,5 & 29,0 \\
\hline Conjoint actif & 62,6 & 62,6 & 61,4 \\
\hline Conjoint inactif & 16,9 & 13,0 & 9,7 \\
\hline \multicolumn{4}{|l|}{ Profession et catégorie socioprofessionnelle } \\
\hline Entrepreneur, artisan, commerçant & 6,0 & 3,9 & 3,9 \\
\hline Cadre et profession intellectuelle & 15,2 & 21,3 & 30,3 \\
\hline Profession intermédiaire & 25,8 & 26,4 & 25,3 \\
\hline Employé & 30,9 & 29,8 & 27,4 \\
\hline Ouvrier & 21,5 & 18,3 & 13,0 \\
\hline \multicolumn{4}{|l|}{ Temps de travail } \\
\hline Temps plein & 86,2 & 82,9 & 86,1 \\
\hline Temps partiel & 13,8 & 17,1 & 13,9 \\
\hline \multicolumn{4}{|l|}{ Lieu de résidence } \\
\hline Centre-ville & 32,0 & 36,5 & 47,8 \\
\hline $1^{\text {re }}$ couronne & 30,7 & 23,8 & 42,0 \\
\hline $2^{\mathrm{e}}$ couronne & 37,3 & 39,7 & 10,2 \\
\hline \multicolumn{4}{|l|}{ Autonomie } \\
\hline Habite chez ses parents & 7,2 & 6,3 & 4,6 \\
\hline \multicolumn{4}{|l|}{ Accessibilité à la voiture } \\
\hline A le permis & 90,7 & 92,5 & 90,4 \\
\hline Nombre de permis/nombre de véhicules dans le ménage & 0,65 & 0,84 & 0,80 \\
\hline Nombre d'observations & 5411 & 5763 & 5328 \\
\hline
\end{tabular}

Lecture : l'échantillon de 1995 était constitué de 5411 individus, parmi lesquels 52,4\% sont des hommes.

Champ : individus en emploi, âgés de 18 à 70 ans, résidant et travaillant dans le périmètre 1995 de l'EMD de Lyon.

Source : EMD de Lyon 1995, 2006, 2015 ; calculs des auteurs.

active en 2017) témoigne de sa bonne santé économique. La progression de l'emploi a été particulièrement soutenue ( $+5,9 \%$ entre 2008 et 2018 , alors que cette croissance est de 3,3 \% sur l'ensemble de la région Auvergne-Rhône-Alpes sur la même période), portée par les activités tertiaires marchandes, tandis que l'emploi industriel continue 
de décliner sur la métropole. L'emploi non salarié est en nette progression $(+36 \%$ entre 2008 et 2018), et s'explique par le développement des micro-entreprises et des professions libérales (MARY-PORTAS, 2018). Ainsi, la proportion de cadres et professions intellectuelles a significativement augmenté entre les enquêtes réalisées en 1995 et 2015 (de 15,2 \% à 30,3\%), alors que la proportion d'ouvriers a fortement diminué sur la même période (de 21,5\% à $13 \%$ ) sur l'ensemble du périmètre.

Par conséquent, il est indispensable dans notre étude de l'évolution temporelle des mobilités domicile-travail d'essayer de dissocier ce qui est attribuable aux changements structurels des caractéristiques des actifs sur le périmètre considéré (évolution des PCS, des lieux de résidence, des situations familiales, etc.) de ce qui est imputable à des modifications de comportements. Pour ce faire, nous avons recours à des modélisations économétriques (cf. infra).

\section{Deux tendances de fond : des distances domicile-travail plus longues et un moindre usage de la voiture}

Nous avons retenu deux indicateurs principaux associés aux déplacements quotidiens domicile-travail : la distance parcourue entre les lieux de résidence et de travail (intra-périmètre) et l'usage de la voiture pour se rendre sur le lieu de travail, qui seront les variables dépendantes dans nos régressions économétriques. Le tableau 2 montre l'évolution de ces variables au cours des vingt dernières années. Pour les individus résidant et travaillant dans l'agglomération lyonnaise, le nombre moyen de déplacements quotidiens en semaine a diminué entre 1995 et 2015 (passant de 4,37 à 3,93) et, parallèlement, les distances moyennes domicile-travail ont augmenté de plus de $20 \%$ (4,46 km en 1995 contre 5,37 km en 2015). Ces résultats correspondent à l'évolution constatée sur l'ensemble du territoire français, puisque le dernier recensement a mis en évidence un allongement des distances parcourues par les actifs pour se rendre au travail (en moyenne 14,6 km en 2013, contre 13 km en 1999) (CoudÈnE, LEVY, 2016). Le fait de conduire sa voiture particulière pour se rendre à son travail a aussi beaucoup évolué en vingt ans : après une légère hausse de l'usage de la voiture entre 1995 et 2006 (+ 3 points de pourcentage), on peut noter une forte baisse entre 2006 et $2015(-8,4$ points de pourcentage).

Plusieurs explications possibles peuvent être avancées de ces récentes tendances, non spécifiques à l'agglomération lyonnaise, d'allongement des distances domiciletravail et du moindre usage de la voiture pour se rendre au travail. Historiquement, les distances parcourues, y compris pour aller travailler, ont augmenté en même temps que la vitesse des déplacements (hausse liée au développement du réseau routier, du réseau ferroviaire, des transports en commun, à la périphérisation de certains flux, etc.), alors que les temps de trajet ne se sont pas forcément allongés. Depuis la fin des années 1990, les phénomènes de densification, de métropolisation et de périurbanisation se 
TABLEAU 2 - Indicateurs de mobilité quotidienne parmi les actifs, selon le genre

\begin{tabular}{|c|c|c|c|c|c|c|c|c|c|}
\hline & \multicolumn{3}{|c|}{ Ensemble } & \multicolumn{3}{|c|}{ Hommes } & \multicolumn{3}{|c|}{ Femmes } \\
\hline & 1995 & 2006 & 2015 & 1995 & 2006 & 2015 & 1995 & 2006 & 2015 \\
\hline \multicolumn{10}{|c|}{ Partie 1 : ensemble de l'échantillon } \\
\hline $\begin{array}{l}\text { Nombre total de } \\
\text { déplacements } \\
\text { (tout motif) }\end{array}$ & 4,37 & $4,14^{* * * *}$ & $3,93^{* * *,+++}$ & 4,36 & $4,02^{* * * *}$ & $3,84^{* * *,+++}$ & 4,38 & $4,26^{*}$ & $4,02^{* * *,+++}$ \\
\hline $\begin{array}{l}\% \text { ne s'étant } \\
\text { pas rendus } \\
\text { au travail }\end{array}$ & $19,1 \%$ & $23,9 \%^{* * *}$ & $17,2 \%^{* * *,++}$ & $14,4 \%$ & $19,2 \%^{* * *}$ & $14,8 \%^{* * *, n . s .}$ & $24,3 \%$ & $28,9 \%^{* * * *}$ & $19,6 \%$ \\
\hline $\begin{array}{l}\text { Distance } \\
\text { domicile-travail } \\
(\text { en } \mathrm{km})^{\mathrm{a}}\end{array}$ & 4,46 & $4,70^{* * * *}$ & $5,37^{* * *,+++}$ & 5,11 & $5,35^{*}$ & $5,87^{* * *,+++}$ & 3,73 & $4,02^{* *}$ & $4,84^{* * * *,+++}$ \\
\hline $\begin{array}{l}\text { Nombre } \\
\text { d'observations }\end{array}$ & 5411 & 5763 & 5328 & 2838 & 2944 & 2702 & 2573 & 2819 & 2626 \\
\hline
\end{tabular}

Partie 2 : échantillon restreint aux individus ayant effectué au moins un déplacement domicile-travail

\begin{tabular}{lccc|ccc|ccc}
\hline $\begin{array}{l}\text { Distance } \\
\text { domicile-travail } \\
\text { (en km) }\end{array}$ & 5,51 & $6,17^{* * *}$ & $6,49^{* * *,+++}$ & 5,98 & $6,62^{* * *}$ & $6,89^{*,+++}$ & 4,93 & $5,64^{* * *}$ & $6,02^{* *,+++}$ \\
\hline $\begin{array}{l}\text { Usage } \\
\text { de la voiture b }\end{array}$ & $68,1 \%$ & $71,3 \%^{* * *}$ & $62,9 \%^{* * *++++}$ & $74,3 \%$ & $76,1 \%^{\text {n.s. }}$ & $66,3 \%^{* * *++++}$ & $59,9 \%$ & $65,6 \%^{* * *}$ & $59,2 \%^{* * *, \text { n... }}$ \\
\hline $\begin{array}{l}\text { Nombre } \\
\text { d'observations }\end{array}$ & 4377 & 4385 & 4412 & 2429 & 2380 & 2301 & 1948 & 2005 & 2111 \\
\hline
\end{tabular}

Notes: ${ }^{a}$ La distance domicile-travail correspond à la distance entre le domicile et le lieu de travail habituel ; les individus n'ayant effectué aucun déplacement domicile-travail la veille de l'enquête ont une distance domicile-travail nulle.

${ }^{\mathrm{b}} \mathrm{La}$ variable usage de la voiture indique le pourcentage des individus qui déclarent utiliser en général une voiture particulière pour se rendre à leur travail.

Les symboles * indiquent la significativité aux tests d'égalité en moyenne effectués entre deux années d'enquêtes consécutives. Les symboles ${ }^{+}$indiquent la significativité aux tests d'égalité en moyenne effectués entre 1995 et $2015 .{ }^{*}$ ou ${ }^{+}$significatif au seuil de $10 \%$, ${ }^{* *}$ ou $^{++}$significatif au seuil de $5 \%,{ }^{* * *}$ ou ${ }^{+++}$significatif au seuil de $1 \%$, n.s. : non significatif.

Champ : individus en emploi, âgés de 18 à 70 ans, résidant et travaillant dans le périmètre 1995 de l'EMD de Lyon.

Source : EMD de Lyon 1995, 2006, 2015 ; calculs des auteurs.

sont combinés au niveau de notre périmètre d'étude (AGENCES D'URBANISME LYON \& SAINT-ÉTIENNE, 2015). L'un des facteurs expliquant la croissance de la population périurbaine est le fort attrait pour la maison individuelle à prix abordable, qui a eu pour conséquence un allongement des temps de parcours domicile-travail. En outre, le foncier se raréfiant dans les centres-villes, la flambée des prix de l'immobilier a aussi pesé sur certaines entreprises - notamment celles cherchant des espaces pour l'industrie et la logistique - qui ont choisi de se relocaliser plus loin du centre. Dans les onze autres métropoles régionales comptabilisées en $2015^{5}$, on observe une même concentration de l'emploi en centre-ville (en niveau) et une dynamique plus soutenue dans les zones périphériques, en raison de la croissance de la population périurbaine et de la recherche d'espaces à proximité des pôles urbains. Grâce notamment à la redensification du

5. Aix-Marseille, Bordeaux, Grenoble, Lille, Montpellier, Nantes, Nice, Rennes, Rouen, Strasbourg et Toulouse. Depuis le $1^{\text {er }}$ janvier 2018, la France compte 22 métropoles (pour plus de détails, voir https://www.cget.gouv.fr/ territoires/metropoles, consulté le 6 juillet 2020). 
centre-ville, expliquée par la présence de certaines catégories socioprofessionnelles et qui contrebalance le phénomène de périurbanisation, la métropole de Lyon affiche une croissance de l'emploi équivalente au sein de la métropole administrative et dans l'aire urbaine ou sa couronne, alors que ce phénomène n'est pas observé dans les autres métropoles (ALTABER, Le HiR, 2017).

L'évolution du pourcentage des actifs occupés qui ne se sont pas rendus sur leur lieu de travail le jour de référence de l'enquête est un autre facteur influençant les distances domicile-travail parcourues, puisqu'en cas de non-déplacement pour motif travail, la distance parcourue reportée est égale à zéro. Selon le tableau 2, la proportion d'actifs ne se rendant pas sur leur lieu de travail est passée de 19,1\% en 1995 à 23,9\% en 2006, puis a baissé pour atteindre 17,2 \% en 2015. L'augmentation entre 1995 et 2006 est probablement en partie attribuable à l'augmentation de la proportion des actifs à temps partiel sur cette période (tableau 1), mais également à la mise en place, à partir du début des années 2000, de la réduction du temps de travail dans le cadre de la loi Aubry I, qui a engendré une réorganisation des journées de travail pour de nombreux individus. Après 2006, la proportion d'individus à temps partiel a diminué ; l'assouplissement de la loi Aubry I et la mise en place à l'été 2007 de la loi en faveur du travail, de l'emploi et du pouvoir d'achat (Tepa) facilitant le recours aux heures supplémentaires pourraient être des éléments restructurant le quotidien et les déplacements pour motif travail.

Par ailleurs, en France comme dans d'autres pays, les études se rejoignent sur le fait que les individus utilisent de moins en moins la voiture particulière, en particulier les jeunes adultes (KUHNIMHOF et al., 2012 ; KUHNIMHOF et al., 2013 ; METZ, 2013 ; WAARD et al., 2013 ; VINCENT et al., 2014). L'urbanisation, le développement des services de transports publics et la diffusion des technologies de l'information et de la communication, associés à la diversification des modes de transport alternatifs (ORTAR et al., 2015), contribuent à expliquer cette tendance, qui tient aussi aux prises de conscience plus marquées des externalités négatives de la voiture (pollution, congestion, nuisances sonores, gaz à effet de serre, accidents de la route, etc.). En outre, les récentes politiques de mobilité mises en œuvre dans les grandes agglomérations (diminution des places de stationnement, quartiers interdits aux véhicules motorisés, réduction du nombre de voies pour les voitures particulières au profit de voies réservées pour les transports en commun, limitation de la vitesse en ville, etc.), s'accompagnant d'une hausse des prix des carburants, ont découragé un certain nombre de personnes d'utiliser leur voiture pour leur mobilité quotidienne (OAKIL et al., 2016). Sur le territoire de l'agglomération lyonnaise, une politique volontariste a été menée depuis les années 2000 pour diminuer la place de la voiture dans les déplacements urbains. Elle s'est notamment traduite par de forts investissements en faveur des transports en commun et une offre plus large de modes de transport doux (encadré 1). 


\section{Changements structurels ou modifications de comportements?}

Il est fort probable que derrière les évolutions globales de ces indicateurs de mobilité se cachent des disparités entre certaines catégories de travailleurs. C'est pourquoi nous avons recours à des modélisations économétriques pour tester l'influence relative de facteurs sociodémographiques (sexe, âge, composition du ménage), économiques (PCS, temps plein/temps partiel), spatiaux (zone de résidence) ou concernant la motorisation (nombre de voitures disponibles par titulaire de permis de conduire du ménage, détention du permis) sur la mobilité quotidienne liée au travail. Parmi les caractéristiques individuelles, nous avons retenu l'âge et le genre. Nous suspectons que l'âge a une influence non linéaire sur le niveau de mobilité, avec un effet positif en début de carrière et un effet plutôt négatif lorsque l'on s'approche de la retraite. Selon la littérature (BACCAÏNI et al., 2007 ; Olmo SANCHEZ, MAESO GonZALEZ, 2016 ; FAN, 2017), nous nous attendons également à ce que la distance parcourue et l'usage de la voiture pour se rendre au travail soient plus élevés chez les hommes que chez les femmes. La composition du ménage a été prise en compte à l'aide de variables combinant statut matrimonial, nombre et âge des enfants, afin de capter les contraintes de gestion des activités domestiques qui influencent les pratiques de mobilité quotidienne, y compris celles pour motif « travail ». De même, nous avons intégré une variable indiquant si l'individu avait ou non un conjoint en emploi et une autre précisant si l'individu habitait encore chez ses parents, cette dernière pouvant aussi capter le poids de contraintes financières.

Nous retenons aussi les caractéristiques de l'emploi occupé : le temps de travail (plein ou partiel) et la PCS. Selon les modèles théoriques, les salariés à temps partiel, percevant en moyenne des rémunérations plus faibles, ont tendance à accepter des emplois davantage situés à proximité de leur domicile que les salariés à temps plein, pour réduire leurs coûts de déplacements, qu'ils soient monétaires ou temporels. De même, les catégories socioprofessionnelles associées aux salaires les plus faibles, telles que les ouvriers, auraient intérêt à opter pour des emplois proches de leur domicile, mais cet effet pourrait être contrebalancé par le choix de localisation de certaines entreprises en périphérie. En outre, les déterminants usuels de la mobilité et de l'usage de la voiture, tels que le lieu de résidence (centre-ville $/ 1^{\text {re }}$ couronne $/ 2^{\mathrm{e}}$ couronne) et l'accessibilité à la voiture particulière, ont également été pris en compte.

Les modèles économétriques - dont les spécifications sont présentées dans l'encadré 2 - sont estimés sur l'échantillon complet, constitué à partir des trois vagues de l'EMD de Lyon. Nous avons introduit, en plus des facteurs évoqués précédemment, deux variables explicatives dichotomiques pour identifier l'année de l'enquête. Les termes d'interaction entre les variables explicatives et ces variables dichotomiques permettent de savoir si les déterminants des variables dépendantes ont changé au cours des périodes étudiées $(1995,2006$ et 2015). Plus précisément, si, pour une variable explicative donnée, le terme d'interaction associé pour 2006 (respectivement 2015) 
n'est pas statistiquement significatif, cela signifie que l'effet de cette variable sur la variable dépendante analysée reste constant entre 1995 et 2006 (respectivement 2015). Si le terme d'interaction est statistiquement significatif et du même signe que l'effet principal de la variable explicative, on peut conclure que son effet a augmenté entre les deux enquêtes. Inversement, si le terme d'interaction est significatif mais de signe opposé à l'effet principal, alors on peut conclure que l'effet de la variable explicative a diminué. Par conséquent, les changements temporels associés à des modifications de comportements seront repérés grâce à la significativité des termes d'interaction. Le tableau en annexe reporte les résultats de nos estimations économétriques. Pour faciliter l'interprétation de nos résultats, le tableau 3 présente les effets marginaux, calculés pour nos deux spécifications et pour nos trois années. Les parties suivantes détaillent les principaux résultats de l'analyse des déterminants des distances domiciletravail puis ceux du recours à la voiture.

\section{ENCADRÉ 2}

\section{Spécifications économétriques}

\section{Distance domicile-travail parcourue}

Pour analyser les distances domicile-travail (dist $)_{i}$, nous proposons d'estimer un modèle Tobit (MADDALA, 1999). Celui-ci permet de tenir compte de la censure à gauche des distributions, liée au fait que des actifs de l'échantillon n'ont effectué aucun déplacement pour motif professionnel la veille du jour de l'entretien d'enquête et ont donc reporté des distances domicile-travail nulles pour ce jour-là. La vraisemblance d'un Tobit est en effet composée d'une fonction des données censurées et d'une fonction conditionnelle des données non censurées (valeur de la distance sachant que l'individu a effectué au moins un déplacement domicile-travail) :

$$
L=\prod_{i=1}^{n}\left\{\left[1-\Phi\left(\frac{X_{i} \beta}{\sigma}\right)\right]^{1-d_{i}} \cdot\left[\varphi\left(\frac{\text { dist }_{i}-X_{i} \beta}{\sigma}\right)\right]^{d_{i}}\right\}
$$

avec $d_{i}$ une variable dichotomique qui vaut 1 si l'individu $i$ a effectué un déplacement domicile-travail la veille et 0 sinon; $\Phi$ et $\varphi$ les fonctions de répartition et de densité respectives d'une loi normale de moyenne nulle et d'écart-type $\sigma$. Les coefficients $\beta$ associés aux variables explicatives $X$ et le paramètre $\sigma$ sont estimés par maximisation de cette fonction de vraisemblance.

\section{Usage de la voiture pour se rendre au travail}

Pour ceux qui ont effectué un déplacement domicile-travail la veille de l'enquête, la probabilité d'avoir ou non utilisé une voiture pour le faire est modélisée par un Logit simple, la variable à expliquer étant dichotomique :

$$
\text { Usgev }_{i}= \begin{cases}1 & \text { Usgev }_{i} \\ 0 & \text { S }_{i} \beta+u_{i}>0, \text { sinon }\end{cases}
$$


où $X_{i}$ est toujours le vecteur de caractéristiques individuelles, $\beta$ les paramètres inconnus captant leur influence et $u$ est un terme d'erreur distribué selon une loi logistique. Les paramètres sont estimés par maximisation de la vraisemblance :

$$
L=\prod_{i=1}^{n}\left\{\left[1-F\left(X_{i} \beta\right)\right]^{1-U_{s g e v}} \cdot\left[F\left(X_{i} \beta\right)\right]^{\text {Usgev }_{i}}\right\},
$$

avec $F$ la fonction de répartition du terme d'erreur $u$.

\section{Limites}

Les Enquêtes ménages déplacements contenant peu de dimensions explicatives, il nous est impossible de proposer des modèles qui prennent explicitement en compte les principaux problèmes d'endogénéité et de biais de sélection. Par exemple, nous avons retenu comme variable explicative de l'usage de la voiture le lieu de résidence de l'individu. Or nous pouvons suspecter que le fait d'habiter en grande couronne oblige l'individu à utiliser sa voiture pour se rendre au travail, faute de transports alternatifs, mais aussi que les individus qui possèdent une voiture et l'utilisent sont davantage prêts à habiter en grande couronne. Ainsi, les décisions peuvent être concomitantes, générant un problème d'endogénéité. Malheureusement, les EMD ne nous permettent pas de mettre en œuvre la technique des variables instrumentales ou d'estimer des systèmes d'équations simultanées appropriés. De même, on peut supposer un problème de biais de sélection en raison de la restriction de notre échantillon à la population en emploi : les personnes en emploi pourraient avoir des caractéristiques inobservables spécifiques qui affectent aussi l'usage de la voiture. Ainsi, nos résultats ne seront pas généralisables à l'ensemble de la population.

\section{Les écarts entre travailleurs dans les distances domicile-travail ont peu évolué entre 1995 et 2015}

Le tableau 3 montre que les facteurs démographiques tels que l'âge, la composition du ménage ou le fait d'habiter chez ses parents ne semblent pas avoir de véritables effets propres sur la distance domicile-travail parcourue. En fait, ce sont les variables relatives au lieu de résidence du ménage et les caractéristiques de l'emploi des répondants (PCS, temps plein/temps partiel) qui sont prédominantes, les variables de motorisation n'arrivant qu'après (encadré 3 ). Or seule l'influence de la localisation géographique et celle de l'accès à la voiture se sont modifiées en vingt ans, les termes d'interaction associés aux autres variables de contrôle n'étant pas significatifs. Les inégalités entre hommes et femmes, entre cadres et ouvriers ou encore entre travailleurs à temps plein et à temps partiel pour les distances domicile-travail sont, elles, restées stables.

Les travailleurs à temps partiel ont, en moyenne, une distance domicile-travail parcourue inférieure de $0,9 \mathrm{~km}$ par rapport à leurs homologues à temps plein, et ce quelle que soit l'année considérée (tableau 3). Ce résultat est dû à deux phénomènes sous-jacents : une distance moyenne entre domicile et lieu d'emploi plus courte pour les travailleurs à temps partiel, mais aussi une proportion plus importante, dans cette catégorie, d'individus ayant une distance parcourue nulle le jour considéré par l'enquête, en raison d'un nombre plus réduit de journées travaillées. En effet, selon nos 
La mobilité domicile-travail des actifs de l'aire urbaine lyonnaise : une approche temporelle

\section{ENCADRÉ 3}

\section{Importance relative des facteurs explicatifs}

Pour classer les caractéristiques selon leur importance, nous avons utilisé le pseudoR2 proposé par Wooldridge, qui mesure le pouvoir prédictif d'un modèle. Pour nos deux spécifications retenues (Tobit pour la distance domicile-travail parcourue et Logit pour l'usage de la voiture pour se rendre au travail), nous avons calculé le pseudo-R2 du modèle complet et celui des modèles où les différentes dimensions ont été enlevées successivement. La dimension la plus influente est celle qui, lorsqu' on la retire du modèle, dégrade le plus la qualité prédictive du modèle, c'est-à-dire provoque la plus forte baisse du pseudo-R2 (AFsA, 2016).

Dans notre cas, nous avons considéré cinq dimensions : motorisation potentielle, lieu de résidence, configuration familiale (composition du ménage, conjoint actif ou non, habiter chez ses parents), caractéristiques de l'emploi (PCS, temps plein/partiel), caractéristiques individuelles (âge, femme).

Le tableau ci-dessous reporte les pseudo-R2 obtenus pour les différentes estimations réalisées sur les données des trois années disponibles.

\begin{tabular}{lcc}
\hline & \multicolumn{2}{c}{ Pseudo-R2 pour le modèle expliquant } \\
\hline Dimension retirée & la distance domicile-travail & l'usage de la voiture \\
\hline Caractéristiques individuelles & 0,0234 & 0,2812 \\
Caractéristiques de l'emploi & 0,0224 & 0,2822 \\
Configuration familiale & 0,0247 & 0,2771 \\
Lieu de résidence & 0,0131 & 0,2566 \\
Motorisation potentielle & 0,0231 & 0,1276
\end{tabular}

estimations, le fait d'être à temps partiel accroît la probabilité de ne pas s'être rendu sur son lieu de travail (c'est-à-dire d'avoir une distance parcourue domicile-travail nulle) de 5,6 points de pourcentage par rapport au fait d'être à temps plein. Parmi les travailleurs ayant une distance parcourue positive, ceux à temps partiel ont effectué des distances domicile-travail inférieures de $0,85 \mathrm{~km}$ à ceux travaillant à temps plein. Ces résultats sont cohérents avec les enseignements de la théorie de recherche d'emploi (BOUABDAlLAH, et al., 2002 ; BRUECKNER, ZENOU, 2003) : les salaires des travailleurs à temps partiel étant plus faibles, travailler à proximité de leur domicile est souvent une condition pour accepter ce type d'emploi, de telle sorte que le revenu net tiré du travail (net des coûts indirects monétaires ou temporels tels que le coût de transport) soit supérieur à leur salaire de réserve (salaire à partir duquel les individus acceptent une proposition d'emploi).

Suivant les mêmes arguments, on s'attendrait à ce que les travailleurs appartenant aux catégories socioprofessionnelles les moins bien rémunérées occupent des emplois plus proches de leur domicile. À partir de statistiques descriptives, on constate, comme l'ont fait de précédentes études, que les cadres parcourent des distances 
TABLEAU 3 - Effets marginaux associés aux modèles économétriques

\begin{tabular}{|c|c|c|c|c|c|c|}
\hline & \multicolumn{3}{|c|}{$\begin{array}{l}\text { Distance domicile-travail } \\
\text { (en km) }\end{array}$} & \multicolumn{3}{|c|}{ Usage de la voiture } \\
\hline & 1995 & 2006 & 2015 & 1995 & 2006 & 2015 \\
\hline Individu de référence & 5,72 & 6,84 & 9,18 & $89,31 \%$ & $91,24 \%$ & $96,53 \%$ \\
\hline Femme & $-0,91$ & $-0,91$ & $-0,91$ & $-9,29$ & $-9,29$ & $-1,07$ \\
\hline Âge & n.s. & n.s. & n.s. & $-0,14$ & $-0,14$ & $-0,14$ \\
\hline \multicolumn{7}{|l|}{ Composition du ménage } \\
\hline Célibataire & Réf. & Réf. & Réf. & Réf. & Réf. & Réf. \\
\hline En couple sans enfant & n.s. & n.s. & n.s. & $+6,10$ & $+2,15$ & $+0,93$ \\
\hline Famille monoparentale & n.s. & n.s. & n.s. & $+8,71$ & $+3,71$ & $+1,81$ \\
\hline Famille 1 enfant, moins de 5 ans & n.s. & n.s. & n.s. & $+6,35$ & $+6,35$ & $+6,35$ \\
\hline Famille 1 enfant, de plus de 5 ans & n.s. & n.s. & n.s. & $+8,06$ & $+3,57$ & $+1,21$ \\
\hline Famille 2 enfants, dont moins de 5 ans & n.s. & n.s. & n.s. & $+8,05$ & $+8,05$ & $+1,51$ \\
\hline Famille 2 enfants, sans moins de 5 ans & n.s. & n.s. & n.s. & $+9,07$ & $+3,48$ & $+1,16$ \\
\hline Famille 3 enfants ou plus, dont moins de 5 ans & n.s. & n.s. & n.s. & $-9,55$ & $+1,65$ & $-9,55$ \\
\hline Famille 3 enfants ou plus, sans moins de 5 ans & n.s. & n.s. & n.s. & $+9,45$ & $+2,00$ & $+1,38$ \\
\hline A un conjoint en emploi & n.s. & n.s. & n.s. & $-3,27$ & $-3,27$ & $-3,27$ \\
\hline \multicolumn{7}{|l|}{ Profession et catégorie socioprofessionnelle } \\
\hline Entrepreneur, artisan, commerçant & $-1,61$ & $-1,61$ & $-1,61$ & n.s. & n.s. & n.s. \\
\hline Cadre et profession intellectuelle & n.s. & n.s. & n.s. & $+2,51$ & $+2,51$ & $-2,12$ \\
\hline Profession intermédiaire & n.s. & n.s. & n.s. & $+3,63$ & $+3,63$ & $-1,46$ \\
\hline Employé & n.s. & n.s. & n.s. & n.s. & n.s. & $-3,64$ \\
\hline Ouvrier & Réf. & Réf. & Réf. & Réf. & Réf. & Réf. \\
\hline \multicolumn{7}{|l|}{ Temps de travail } \\
\hline Temps plein & Réf. & Réf. & Réf. & Réf. & Réf. & Réf. \\
\hline Temps partiel & $-0,87$ & $-0,87$ & $-0,87$ & n.s. & n.s. & n.s. \\
\hline \multicolumn{7}{|l|}{ Lieu de résidence } \\
\hline Centre-ville & $-2,13$ & $-2,88$ & $-4,46$ & $-8,95$ & $-19,60$ & $-22,27$ \\
\hline $1^{\mathrm{re}}$ couronne & $-1,50$ & $-2,17$ & $-3,14$ & n.s. & $-5,49$ & $-7,63$ \\
\hline $2^{e}$ couronne & Réf. & Réf. & Réf. & Réf. & Réf. & Réf. \\
\hline Habite chez ses parents & n.s. & n.s. & n.s. & $-9,06$ & $-9,06$ & $-9,06$ \\
\hline Accessibilité à la voiture & $+0,41$ & $+0,41$ & $+1,56$ & $+39,29$ & $+8,32$ & $+3,37$ \\
\hline Permis & $+0,78$ & $+0,78$ & $+0,78$ & & & \\
\hline Nombre d'observations & & 16437 & & & 12527 & \\
\hline
\end{tabular}

Notes : la première ligne du tableau indique les valeurs estimées pour l'individu de référence, à savoir un homme célibataire, sans enfant, de 41 ans, occupant un emploi d'ouvrier à temps plein et résidant en deuxième couronne et n'ayant pas le permis. Les effets marginaux correspondent à la variation de ces valeurs prédites quand on modifie l'une de ces caractéristiques. n.s. : écart non significatif à un seuil de $5 \%$ par rapport à la catégorie de référence.

Lecture : toutes choses étant égales par ailleurs, la distance domicile-travail effectuée par les femmes le jour de référence de l'enquête est inférieure de 0,91 km en moyenne par rapport à celle de leurs homologues masculins, sur les trois années étudiées $(1995,2006,2015)$. De plus, la probabilité qu'ont les femmes d'utiliser la voiture pour se rendre au travail est inférieure de 9,29 points de pourcentage par rapport aux hommes en 1995 et 2006 et inférieure de seulement 1,07 point de pourcentage en 2015.

Champ : individus en emploi, âgés de 18 à 70 ans, résidant et travaillant dans le périmètre 1995 de l'EMD de Lyon.

Source : EMD de Lyon 1995, 2006, 2015 ; calculs des auteurs.

domicile-travail plus grandes que les autres catégories de salariés (BACCAÏNI et al., 2007 ; BERGER, 2004) : cet écart est de l'ordre de 0,7 km en moyenne sur l'ensemble de nos trois enquêtes, ce qui est un peu plus faible que dans la littérature et s'explique 
peut-être par notre échantillon, qui porte uniquement sur les individus résidant et travaillant dans le périmètre de l'EMD 1995. Pour des questions de comparabilité des données des différentes vagues d'enquête, nous avons en effet exclu de notre analyse les individus qui effectuent des mobilités professionnelles longue distance. D'après une publication de l'OBSERVATOIRE PARTENARIAL DÉPLACEMENTS de l'Agence d'urbanisme pour le développement de l'agglomération lyonnaise (2012), un actif occupé sur dix résidant dans la communauté urbaine travaille en dehors de ce territoire. Or ces navetteurs sont beaucoup plus nombreux parmi les cadres que parmi les autres professions et catégories socioprofessionnelles, ce qui tire vers le haut la moyenne de leurs distances domicile-travail dans les enquêtes les prenant en compte. Notre régression économétrique fait apparaître que l'effet de la PCS sur la distance domiciletravail déclarée est non significatif, à l'exception de celui associé aux entrepreneurs et artisans (tableau 3). Ces résultats ne sont pas contradictoires avec ceux suggérés par les statistiques descriptives. Ils révèlent simplement que les cadres, davantage masculins, possédant plus souvent le permis de conduire, ayant une meilleure accessibilité à la motorisation au sein de leur ménage et travaillant moins à temps partiel que les autres PCS, effectuent des distances domicile-travail plus élevées. Cependant, toutes choses étant égales par ailleurs, les distances domicile-travail parcourues entre un cadre et un individu d'une autre profession qui présenterait exactement les mêmes caractéristiques ne sont guère différentes. En d'autres termes, les écarts observés dans les distances effectives entre ouvriers et cadres par exemple ne sont pas attribuables à la PCS en tant que telle mais au fait que leurs autres caractéristiques sont hétérogènes. Les entrepreneurs et les artisans déclarent en moyenne une distance domicile-travail inférieure de 1,61 km à celle déclarée par les autres PCS. Cette spécificité est en partie attribuable au fait qu'une proportion significativement plus importante d'entre eux que la moyenne $(+4 \%)$ n'effectue aucun déplacement domicile-travail. Par ailleurs, beaucoup d'artisans travaillent et résident dans des locaux non disjoints.

Le tableau 3 met aussi en évidence que, toutes choses étant égales par ailleurs, les femmes parcourent des distances plus courtes que les hommes pour se rendre sur leur lieu de travail : moins $1 \mathrm{~km}$ en moyenne, quelle que soit l'année considérée. Cet effet de genre persistant n'est pas spécifique à l'agglomération lyonnaise, puisqu'il a été reporté à de nombreuses reprises dans les travaux étudiant la mobilité des personnes en emploi en France (BACCAÏNI et al., 2007 au niveau national ; BACCAÏNI, 1996 et SARI, 2011 en région parisienne ; ANDAN et al., 1999 en région lyonnaise) et à l'étranger (Best, Lanzendorf, 2005 ; Kwan, Kotsev, 2015 ; Olmo Sanchez, Maeso GonZalez, 2016 ; FAN, 2017). Ici, les différences de genre observées ne peuvent pas être attribuées au fait que les femmes travaillent davantage à temps partiel, comme certains l'ont fait dans la littérature (HANSON, JOHNSTON, 1985), puisque ce facteur est contrôlé dans notre modèle économétrique explicatif des distances domicile-travail. Certes, le temps partiel plus fréquent chez les femmes ${ }^{6}$ explique en partie les écarts entre

6. En 1995, le temps partiel concernait 24,6\% des femmes contre seulement $4 \%$ des hommes, dans notre échantillon. Un important écart persistait encore en 2015 avec 22,5\% des femmes en temps partiel et 5,5\% des hommes. 
genre dans les distances moyennes domicile-travail observées dans les statistiques descriptives (tableau 2). Mais les résultats de la régression économétrique (tableau 3) indiquent qu'une fois cet effet contrôlé, il existe encore une différence significative en ce qui concerne les distances domicile-travail entre les hommes et les femmes : d'autres facteurs que le temps de travail en sont donc à l'origine. L'explication la plus convaincante de ce phénomène provient probablement de la division des rôles sur le marché du travail et au sein des ménages. En France comme ailleurs en Europe, la répartition des responsabilités domestiques est très liée au genre, les femmes consacrant davantage de temps que les hommes à s'occuper de l'entretien du logement, des enfants et des adultes à charge, en assurant notamment leurs déplacements (McGuCKIN, Murakami, 1999 ; Scheiner, Holz-Rau, 2012 ; Craig, van Tienoven, 2019). Il en résulte que le temps passé dans les transports est souvent beaucoup plus pénalisant pour les femmes. Davantage contraintes par le temps que les hommes, opter pour des emplois plus proches de leur domicile peut être pour ces dernières un choix rationnel pour mieux concilier vie professionnelle et responsabilités familiales. En outre, comme les salaires des femmes continuent d'être plus faibles que ceux de leurs homologues masculins (Minni, 2012 ; Chamkhi, Toutlemonde, 2015 ; Dupray, Moullet, 2015), le bassin d'emplois où leur revenu net (notamment net des coûts de déplacements) est supérieur à leur salaire de réserve est plus restreint autour de leur domicile que celui des hommes. Pour toutes ces raisons, les emplois des femmes sont davantage localisés à proximité de leur lieu de résidence que ceux des hommes, et nous n'observons aucun changement dans l'ampleur de ce phénomène entre 1995 et 2015.

Tout comme pour les caractéristiques de l'emploi ou le genre, l'influence de la détention du permis de conduire sur la distance domicile-travail a peu évolué en vingt ans. En 1995, 2006 et 2015, les actifs occupés détenteurs d'un permis de conduire parcourent en moyenne, pour aller sur leur lieu de travail, une distance supérieure de $0,78 \mathrm{~km}$ aux autres, à caractéristiques contrôlées équivalentes (tableau 3). Si le permis de conduire s'accompagne d'un accès à la voiture particulière, les actifs peuvent atteindre des zones d'emplois plus éloignées de leur lieu de résidence et l'impact sur la distance domicile-travail est plus important (1,2 km supplémentaire en 1995 et 2006 et 2,3 km supplémentaires en 2015), confirmant les résultats de Marlon Boarnet et Randall Crane (2001) et de Frans M. Dieleman et de ses co-auteurs (2002). Nous suspectons ainsi qu'avoir ou non la possibilité de conduire pèse de plus en plus dans les chances d'accéder à un emploi, notamment lorsqu'il est peu qualifié : dans l'agglomération lyonnaise en effet, la croissance dynamique de ces emplois a été marquée par la périurbanisation, notamment dans les secteurs de l'industrie et des services administratifs. Or ces zones périurbaines sont mal desservies par les transports collectifs, dont l'organisation est essentiellement radiale, et l'accès à ces lieux se fait encore quasi exclusivement en voiture (Bouzouina et al., 2014).

Comme attendu, la localisation géographique du lieu de résidence est le facteur prépondérant dans les déplacements quotidiens domicile-travail, mais son impact a fortement évolué depuis 1995, contrairement à la majorité des autres facteurs. Les 
statistiques descriptives mettent en évidence une relocalisation des actifs au centre de l'agglomération, qui s'explique notamment par une densification de l'aire urbaine. Les actifs résidant en centre-ville ont progressivement modifié leurs comportements de mobilité liée au travail, en réduisant la distance parcourue et en utilisant moins la voiture particulière : alors qu'en 1995, ils parcouraient en moyenne $2,1 \mathrm{~km}$ de moins pour se rendre au travail que les habitants de la grande périphérie (deuxième couronne), l'écart s'est creusé pour atteindre 4,5 km en 2015, toutes choses étant égales par ailleurs. L'évolution est moins sensible si on compare les résidents du centre-ville à ceux de la proche banlieue :-1,5 km en 1995 et -3,1 km en 2015 (tableau 3). Plus généralement, plus le lieu de résidence est éloigné du centre de l'agglomération et plus la distance domicile-travail augmente. Dans les métropoles françaises, les actifs résidant dans les pôles urbains travaillent plus près de chez eux que les actifs habitant en périphérie des villes (BACCAÏNI et al., 2007), du fait notamment des opportunités d'emplois plus importantes en centre-ville. La structure monocentrique de la ville de Lyon et le réseau de transports en commun développé « en étoile » contribuent à expliquer ce phénomène, qui s'est considérablement accentué entre 1995 et 2015 (BoNNEL et al., 2013). Selon une publication de l'OBSERVATOIRE PARTENARIAL DÉPLACEMENTS de l'Agence d'urbanisme pour le développement de l'agglomération lyonnaise (2012), les communes de Lyon et de Villeurbanne, qui constituent le centre dense, regroupent non seulement la plus forte part des emplois de l'agglomération, mais également la plus forte proportion de salariés qui y résident. À l'inverse, les actifs habitant en périphérie des pôles urbains travaillent plus rarement à proximité de leur domicile, ce qui augmente les distances parcourues pour se rendre sur leur lieu de travail.

Notre régression sur la distance domicile-travail parcourue confirme que l'évolution de cet indicateur, observée entre 1995 et 2015, n'est pas entièrement imputable aux changements de caractéristiques de la population au sein du périmètre étudié. Certains actifs, en particulier les habitants des centres-villes, semblent avoir modifié leurs pratiques de mobilité. Ce phénomène est encore plus flagrant quand on s'intéresse au mode de transport utilisé pour aller travailler.

\section{Des changements de comportements vis-à-vis de l'usage de la voiture en vingt ans}

Sans surprise, le degré d'accessibilité à une voiture, mesuré par le nombre de voitures disponibles dans le ménage par titulaire du permis, est la caractéristique la plus déterminante dans l'usage de la voiture pour les actifs en emploi, avant le lieu de résidence et la configuration familiale, tandis que les caractéristiques de l'emploi et de l'individu n'arrivent qu'après (encadré 3). Plus le ménage de l'individu possède de voitures, plus la probabilité de les utiliser pour se rendre au travail est élevée. Toutefois, le tableau 3 nous montre que l'effet marginal associé à cette variable baisse au fil des années. Cela signifie que l'accès potentiel à une voiture est de moins en moins synonyme de son utilisation systématique pour aller travailler. En fait, l'influence de 
l'ensemble des déterminants de l'utilisation quotidienne de la voiture particulière (à l'exception de l'âge, du fait d'avoir un conjoint en emploi et du fait d'habiter chez ses parents), qu'ils soient plus ou moins prépondérants, a évolué au cours de la période étudiée, alors que l'on ne trouvait rien de tel dans l'analyse des distances domiciletravail. La baisse générale de l'usage de la voiture serait ainsi le reflet de véritables modifications des pratiques de mobilité.

Le lieu de résidence impacte également fortement l'utilisation quotidienne de la voiture particulière. En 1995, les actifs du centre-ville avaient une probabilité inférieure de 8,9 points (tableau 3 ) de pourcentage d'utiliser ce moyen de transport pour se rendre sur leur lieu de travail que ceux de la périphérie. Cette probabilité est inférieure de plus de 19 points de pourcentage en 2006 et de 22 points de pourcentage en 2015. Depuis 2006 , les actifs habitant en proche banlieue (première couronne) sont également moins dépendants de la voiture pour aller travailler que ceux de la deuxième couronne (probabilité inférieure de 5,5 points de pourcentage en 2006 et de 7,6 points de pourcentage en 2015), mais dans une moindre mesure par rapport à ceux du centre-ville. Ceux qui résident en périphérie (première et deuxième couronnes) sont davantage motorisés, et utilisent plus souvent la voiture pour accéder à un pôle d'emplois plus vaste, souvent plus éloigné de leur lieu de résidence, contrairement aux actifs du centre, qui peuvent facilement substituer à l'usage de la voiture celui des transports en commun ou des modes de transports dits « doux » (marche à pied, vélo, trottinette, etc.) La politique volontariste en vigueur depuis les années 2000 dans l'agglomération lyonnaise pour diminuer la place de la voiture a en priorité touché les actifs résidant en centre-ville. Elle a ensuite permis, certes dans une moindre mesure, de diminuer l'usage de la voiture des actifs résidant en proche périphérie (première couronne), grâce notamment au développement d'infrastructures de transports en commun. En effet, alors que de nombreux modes de transports sont mis à disposition des actifs, et plus largement des habitants, la métropole lyonnaise cherche, dans le cadre de sa stratégie « Métropole intelligente ${ }^{7} »$, à faire évoluer les comportements et à fluidifier et réduire le trafic automobile. Dans ce cadre, elle soutient le développement des déplacements doux (Vélo'v), des solutions d'autopartage (Citiz LPA, Bluely, etc.) et de covoiturage, ainsi que celui de nouveaux services destinés à faciliter la mobilité tel Onlymoov, site internet permettant le calcul d'itinéraires personnalisés et la diffusion d'informations trafic en temps réel. Le réseau de transports collectifs a bénéficié d'une amélioration continue des infrastructures, facilitant les déplacements vers les communes de la première couronne ${ }^{8}$.

Toutefois, l'évolution de l'usage de la voiture n'a absolument pas été la même pour l'ensemble des actifs, étant notamment très hétérogène selon la PCS. En 1995 et 2006, les cadres et les professions intermédiaires avaient en moyenne une probabilité d'utiliser la voiture pour se rendre au travail supérieure, respectivement, de 2,5 et

7. Pour en savoir plus : https://www.grandlyon.com/metropole/deplacements.html, consulté le 7 juillet 2020.

8. Prolongement de la ligne de métro A à Vaulx-en-Velin en 2007, de la ligne de métro B en 2013 à Oullins, mise en place de la ligne de tram T4 pour relier le sud (Feyzin) et le nord (La Doua) de l'agglomération en 2009, etc. 
3,6 points de pourcentage à celle des ouvriers. En 2015, la tendance est inverse : la probabilité pour les cadres d'utiliser la voiture est inférieure à celle des ouvriers de 2,1 points de pourcentage, et de 1,5 point pour les professions intermédiaires. Les ouvriers conduisent davantage leur voiture particulière qu'auparavant : $63 \%$ des ouvriers se rendent au travail en voiture en 1995 contre $67 \%$ en 2015. Mais les changements observés quant à l'usage de la voiture ne peuvent pas se justifier seulement par une moindre appétence des salariés les moins qualifiés à changer leurs pratiques de mobilité. Ils relèvent aussi de facteurs liés aux emplois et à leur accessibilité. Alors que les cadres et les professions intermédiaires ont choisi de moins utiliser la voiture, les ouvriers et les employés ont été contraints d'en intensifier leur usage. En effet, comme évoqué précédemment, les premiers travaillent pour beaucoup dans le tertiaire et bénéficient d'une bonne accessibilité en transports collectifs à leur lieu d'emploi, contrairement aux ouvriers et employés, dont les lieux de travail ont souvent été relocalisés en zones périurbaines, mal desservies (Bouzouina et al., 2014). Nos régressions ne permettent pas de contrôler l'accessibilité en transports en commun du lieu de travail, nous captons donc cet effet partiellement, par la PCS. Avec la hausse importante du prix des carburants, certains ouvriers auraient sans doute préféré pouvoir se reporter sur des modes alternatifs à la voiture, dont l'utilisation quotidienne impacte lourdement leur budget. En 2011, les transports étaient d'ailleurs un gros poste de dépenses chez les ouvriers. En particulier, les frais d'utilisation de véhicules (entretien, essence, garagiste, parking, péage) représentaient 8,6\% de leurs dépenses totales, contre seulement $5,8 \%$ chez les cadres (BURON et al., 2014).

Les facteurs démographiques tels que l'âge, la composition du ménage ou le fait d'habiter chez ses parents ont des effets significatifs sur l'usage de la voiture, alors qu'ils semblent peu affecter la distance domicile-travail parcourue. Selon le tableau 3, la probabilité d'aller travailler en voiture des jeunes actifs habitant chez leurs parents est inférieure de 9 points de pourcentage, quelle que soit l'année considérée, à celle des actifs qui ont leur propre logement. Étant donné qu'une grande majorité d'entre eux restent probablement chez leurs parents pour des raisons financières, il est vraisemblable qu'ils considèrent les coûts associés à l'usage de la voiture particulière (passage du permis, achat, assurance, carburant, stationnement) trop élevés (DEMOLI, 2017). La probabilité d'utiliser la voiture pour se rendre sur son lieu de travail est, toutes choses étant égales par ailleurs, concave en fonction de l'âge. Elle est croissante jusqu'à l'âge de 37 ans puis décroissante si l'individu reste célibataire et sans enfant. Notre régression montre que la présence d'enfants influence fortement et significativement le recours à ce mode de transport. En 1995, la probabilité d'utiliser la voiture des actifs célibataires est inférieure de 6 à 9 points de pourcentage par rapport aux actifs ayant une famille avec enfants à charge, à l'exception de ceux qui appartiennent à des familles de trois enfants et plus, dont certains en bas âge (moins de 5 ans). Les actifs appartenant à ces ménages sont en effet ceux qui utilisent le moins la voiture pour se rendre au travail. L'âge des enfants semble jouer puisque les actifs appartenant à une famille avec au moins deux enfants, tous âgés de plus de 5 ans, sont les plus 
susceptibles d'utiliser une voiture particulière pour se rendre à leur travail. On peut supposer que pour ces actifs, ce mode de transport offre une plus grande souplesse pour la gestion parallèle des trajets non liés au travail (accompagner les enfants à leur établissement scolaire ou à leurs activités de loisirs, faire les courses, effectuer des démarches administratives, etc.). Toutefois, les différences dans l'usage de la voiture entre les célibataires et les autres types de ménages se sont très fortement atténuées entre 1995 et 2015. Les actifs appartenant à une famille avec un enfant de moins de 5 ans sont les seuls qui ne semblent pas avoir profondément modifié (à la baisse) leur usage de la voiture sur les vingt dernières années. Pour les autres, et quelle que soit la composition du ménage, la probabilité de se rendre au travail en voiture a diminué, par rapport aux célibataires.

Si l'impact du genre sur les distances domicile-travail est stable entre 1995 et 2015, on observe que l'écart femmes hommes en ce qui concerne le recours à la voiture particulière pour se rendre au travail a fortement diminué. Historiquement, les femmes étaient moins nombreuses à posséder le permis de conduire et celles qui le détenaient avaient un accès plus restreint aux modes de transport motorisés, en particulier à la voiture particulière. C'est pourquoi les femmes actives utilisaient moins souvent et surtout moins régulièrement que les hommes la voiture pour aller travailler (probabilité inférieure de 9,3 points de pourcentage en 1995 et 2006, à autres caractéristiques équivalentes). Mais, depuis 2015, les femmes ont des pratiques modales qui se rapprochent de celles des hommes. Leur probabilité d'utiliser la voiture pour se rendre au travail est inférieure de seulement un point de pourcentage à celle de leurs homologues masculins. La convergence des mobilités entre hommes et femmes semble donc d'abord s'effectuer par le choix du mode de transport. La convergence entre genre des distances parcourues prendra sans doute plus de temps, puisqu'elle ne pourra se faire qu'au prix d'une répartition plus égalitaire des activités de production domestique et d'une réduction des disparités femmes hommes sur le marché du travail.

Notre article étudie l'évolution de la mobilité quotidienne domicile-travail au cours des vingt dernières années dans l'agglomération lyonnaise. Pour ce faire, nous avons utilisé les trois dernières vagues des Enquêtes ménages déplacements de Lyon (1995, 2006 et 2015). En raisonnant à périmètre constant, ces enquêtes permettent de considérer un échantillon représentatif du territoire étudié et d'analyser plusieurs indicateurs de mobilité : la distance domicile-travail et l'usage de la voiture pour se rendre au travail. Sur la période 1995-2015, ces indicateurs ont connu des variations importantes. Les distances domicile-travail (calculées uniquement pour les actifs qui résident et travaillent dans le périmètre de l'enquête 1995) ont augmenté, alors que la proportion d'actifs utilisant leur voiture pour se rendre sur leur lieu de travail a considérablement diminué. Nos régressions économétriques montrent que ces évolutions s'expliquent non seulement par un changement des caractéristiques socioéconomiques 
et des choix de localisation des répondants ${ }^{9}$, mais également par une modification des pratiques de mobilité liées au travail.

Les changements de comportements ont davantage concerné le mode de transport utilisé que la distance domicile-travail effectuée. L'augmentation tendancielle de cette distance a globalement touché de manière similaire l'ensemble des actifs, hormis ceux résidant en centre-ville, pour lesquels la hausse a été beaucoup plus modérée, et pour les actifs utilisant la voiture pour aller travailler, pour lesquels elle a été plus marquée. La forte baisse de l'usage de la voiture pour se rendre au travail s'explique en partie par la politique volontariste des élus de réduire la place de la voiture dans les déplacements intra-urbains, avec une offre de transports en commun qui s'est considérablement étoffée entre 1995 et 2015 dans l'agglomération lyonnaise. De récentes études menées sur le territoire lyonnais montrent que les jeunes adultes, qui se localisent davantage au centre de l'agglomération, sont de moins en moins intéressés par le passage du permis de conduire et l'accès à la voiture particulière (VINCENT et al., 2014). Ainsi, la redensification du centre de l'agglomération par cette population a accentué le recours à des modes alternatifs (transports en commun, modes doux).

Notre étude délivre également des enseignements sur les inégalités en matière de mobilité. En effet, les changements de comportements vis-à-vis de l'usage de la voiture diffèrent fortement selon les catégories socioprofessionnelles et le genre. En particulier, nous avons pu observer que les cadres et les professions intermédiaires, qui utilisaient plus la voiture que les ouvriers en 1995, toutes choses étant égales par ailleurs, y ont davantage renoncé en vingt ans, au point de représenter la catégorie la moins utilisatrice de la voiture en 2015, derrière les employés. Au contraire, les ouvriers n'ont pas réduit leur usage de la voiture, ce qui résulte probablement davantage des contraintes spatiales liées à leur lieu de résidence et de travail qu'à un choix délibéré. Ils occupent en effet plus souvent des emplois en périphérie que les autres, dans des zones moins bien desservies par les transports en commun et subiraient de ce fait le choix de leur mode de transport.

Par conséquent, les différences de pratiques de mobilité entre catégories socioprofessionnelles pourraient être à l'origine d'un renforcement des inégalités sociales. Face au prix croissant des loyers en centre-ville, de nombreuses entreprises, notamment industrielles, s'installent en banlieue ou en proche périphérie. Les tendances constatées dans cette étude, concernant les distances domicile-travail et l'usage de la voiture pour se rendre sur son lieu de travail, devraient donc se poursuivre dans les prochaines années. L'obligation réglementaire depuis le $1^{\mathrm{er}}$ janvier $2018^{10}$, pour les entreprises regroupant plus de 100 salariés sur un même site, d'élaborer des plans de mobilité pour améliorer la mobilité de leur personnel et encourager l'utilisation de transports en commun et le recours au covoiturage, pourrait peut-être changer la donne.

9. Par exemple, de nombreux jeunes adultes et actifs aux emplois qualifiés ont contribué à la redensification du centre de l'agglomération en choisissant d'y habiter.

10. Article 51 de la loi de transition énergétique pour la croissance verte (LTECV). 
Nos résultats mettent aussi en évidence une diminution des effets de genre. Les modes de transport empruntés par les femmes actives se rapprochent en effet de plus en plus de ceux des hommes : cette tendance devrait se poursuivre, favorisée par les politiques de l'emploi qui encouragent l'égalité entre les femmes et les hommes sur le marché du travail. Toutefois, les emplois ne sont toujours pas répartis de manière identique entre les femmes et les hommes, puisque les femmes occupent plus souvent des emplois moins qualifiés et/ou à temps partiel que les hommes. Il est donc vraisemblable que des différences de mobilité liées au travail persisteront tant qu'il y aura des différences dans les postes et les types d'emploi occupés et dans la gestion des tâches domestiques. Là encore, l'obligation réglementaire des plans de mobilité pourrait être l'occasion pour les entreprises de se saisir de ces thématiques pour effectuer un diagnostic précis et construire de véritables projets, améliorant la qualité de vie au travail et réduisant certaines inégalités.

Cette étude a entrouvert la question de la complexité des liens qui existent et évoluent entre mobilité quotidienne et emplois. Comme beaucoup d'analyses de ce type, elle souffre probablement de limites dues à des problèmes d'endogénéité (pour le lieu de résidence ou la motorisation potentielle) et de biais de sélection (l'échantillon est restreint à la population en emploi). Ces problèmes n'ont toutefois pas pu être traités, faute de données adéquates. Ces questions mériteraient d'être approfondies par de futures recherches. Seule l'exploitation de bases de données au niveau individuel, représentatives de la population française, qui contiennent des informations fines et faisant le lien entre la situation vis-à-vis du marché du travail, les lieux de résidence et d'emploi et les principaux indicateurs de mobilité quotidienne, permettrait de contourner ces limites.

Enfin, nos travaux concernent l'agglomération lyonnaise. Il serait intéressant de pouvoir reproduire ces analyses sur d'autres métropoles présentant des configurations et évolutions différentes en matière de structure et de répartition de population et d'emploi, afin d'examiner leur potentiel de transférabilité spatiale.

\section{BibLIOGRAPHIE}

AgenCes D'urbanisme Lyon \& SAINT-ÉTIENNE (2015), Dynamiques de métropolisation. Repères, Lyon, Saint-Étienne, Agences d'urbanisme de Lyon \& Saint-Étienne.

Altaber C., Le Hir B. (2017), « Dynamique de l'emploi dans les métropoles et les territoires avoisinants », La Note d'analyse, France Stratégie, $\mathrm{n}^{\circ} 64$.

Andan O., Pochet P., Routhier J.-L., SÉchou B. (1999), Stratégies de localisation résidentielle des ménages et mobilité domicile-travail, Rapport final pour le compte de la Drast (Predit 1996-2000), Lyon, Laboratoire d'économie des transports.

ASFA C. (2016), « Le modèle Logit : théorie et application », Document de travail, Insee, série «Méthodologie statistique », M2016/01. 
La mobilité domicile-travail des actifs de l'aire urbaine lyonnaise : une approche temporelle (1995-2015)

BACCAÏNI B. (1996), « Les trajets domicile-travail en Île-de-France. Contrastes entre catégories socioprofessionnelles », Économie et Statistique, n ${ }^{\circ}$ 294-295, pp. 109-126.

BACCAÏNI B., SÉMÉCURBE F., ThOMAS G. (2007), « Les déplacements domicile-travail amplifiés par la périurbanisation », Insee première, $\mathrm{n}^{0} 1129$.

BERGER M. (2004), « Mobilités résidentielles, mobilités quotidiennes : une approche des déterminants sociaux des aires de déplacement en région parisienne », communication, Colloque ESO, Rennes, octobre.

Best H., LANZENDORF M. (2005), "Division of Labour and Gender Differences in Metropolitan Car Use: An Empirical Study in Cologne, Germany”, Journal of Transport Geography, vol. 13, $\mathrm{n}^{\mathrm{o}} 2$, pp. 109-121.

BoARnet M., CRANE R. (2001), "The Influence of Land Use on Travel Behavior: Specification and Estimation Strategies", Transport Research Part A: Policy and Practice, vol. 35, $\mathrm{n}^{\circ} 9$, pp. 823-845.

Bonnel P., Bouzouina L., Monchambert G. (2013), « Croissance urbaine et accessibilité : intérêts et limites de l'approche par les densités dans une perspective de modélisation de l'usage du sol », Cybergeo: European Journal of Geography [en ligne], document 642. https://doi. org/10.4000/cybergeo.25892.

Bonnel P., CABAnne I., Massot M.-H. (dir.) (2003), Évolution de l'usage des transports collectifs et politiques de déplacements urbains, Paris, La Documentation française.

Bouabdallah K., Cavaco S., Lesueur J.-Y. (2002), « Recherche d'emploi, contraintes spatiales et durée de chômage : une analyse microéconométrique », Revue d'économie politique, vol. $112, \mathrm{n}^{\circ} 1$, pp. 137-156.

Bouzouina L., Cabrera Delgado J., Emmerich G. (2014), «Inégalités d'accessibilité à l'emploi en transport collectif urbain : deux décennies d'évolutions en banlieue lyonnaise », Revue d'économie régionale et urbaine, vol. 2014, nº 1, pp. 33-61.

BRUECKNER J. K., Zenou Y. (2003), "Space and Unemployment: The Labor-Market Effects of Spatial Mismatch”, Journal of Labor Economics, vol. 21, n 1, pp. 242-266.

Buron M.-L., Kranklader É., Ribera J. (2014), « Le budget des familles en 2011 : analyse et commentaire des principaux résultats de l'enquête "Budget de famille" de 2011 », in Insee Résultats, $\mathrm{n}^{\circ} 158$.

Cabrera Delgado J., Bonnel P. (2016), "Level of Aggregation of Zoning and Temporal Transferability of the Gravity Distribution Model: The Case of Lyon”, Journal of Transport Geography, vol. 51, pp. 17-26.

CENTRE D'ÉTUdES SUR LES RÉSEAUX, LES TRANSPORTS, L'URBANISME ET LES CONSTRUCTIONS PUBLIQUES (CERTU) (2008), L'Enquête ménages déplacements « standard Certu », Lyon, Certu.

Cete de LyOn (2011), Analyse des EDVM réalisées sur la région. Sous rapport, Bron, Cete de Lyon [en ligne] : http://www.bourgogne-franche-comte.developpement-durable.gouv.fr/IMG/ pdf/CETE_DMOB_20110323_Srapport_EDVM_cle722631.pdf, consulté le 16 juillet 2020.

Chamkhi A., Toutlemonde F. (2015), «Ségrégation professionnelle et écarts de salaire femmes-hommes », Dares analyses, $n^{\circ} 082$. 
CoudÈne M., Levy D. (2016), « De plus en plus de personnes travaillent en dehors de leur commune de résidence », Insee première, $\mathrm{n}^{\circ} 1605$.

Craig L., van Tienoven T. P. (2019), "Gender, Mobility and Parental Shares of Daily Travel With and For Children: A Cross-National Time Use Comparison”, Journal of Transport Geography, vol. 76, pp. 93-102.

Dargay J., Gately D., Sommer M. (2007), "Vehicle Ownership and Income Growth, Worldwide: 1960-2030", The Energy Journal, vol. 28, n 4, pp. 143-170.

Dieleman F. M., Dijst M., Burghouwt G. (2002), "Urban Form and Travel Behaviour: MicroLevel Household Attributes and Residential Context”, Urban Studies, vol. 39, n 3, pp. 507-527.

Demoli Y. (2017), «Les jeunes et la voiture, un désir contrarié ?», Métropolitiques.eu [en ligne], 9 octobre : https://www.metropolitiques.eu/Les-jeunes-et-la-voiture-un-desir-contrarie. html, consulté le 16 juillet 2020.

Dupray A., Moullet S. (2015), «Le salaire moindre des femmes : une question d'individu ou de profession? », Travail et Emploi, n ${ }^{\circ} 144$, pp. 81-107.

FAN Y. (2017), "Household Structure and Gender Differences in Travel Time: Spouse/Partner Presence, Parenthood, and Breadwinner Status", Transportation, vol. 44, n 2, pp. 271-291.

GeyiK M., Bonnel P. (2016), Évolution de l'usage des modes de transport. Analyse des facteurs explicatifs : application à l'agglomération lyonnaise, Predit groupe 06, Projet EvolMob, Rapport final, Lyon, Laboratoire aménagement économie transports.

GOYOn M., LeJOUX P., ORTAR N. (2010), Dépendance automobile et territoires périurbains : de nouvelles perspectives?, Rapport final pour l'ADEME.

HANson S., Johnston I. (1985), “Gender Differences in Work-Trip Length: Explanations and Implications", Urban Geography, vol. 6, n 3, pp. 193-219.

Institut NATIONAL DE LA STATISTIQUE ET DES ÉTUdES ÉCONOMiQUES (InSEE) (2019), Tableaux de l'économie française, édition 2019, Paris, Insee, coll. « Insee Références ».

Kuhnimhof T., Armoogum J., Buehler R., Dargay J., Denstadli J. M., Yamamoto T. (2012), "Men Shape a Downward Trend in Car Use among Young Adults - Evidence from Six Industrialized Countries", Transport Reviews, vol. 32, n 6, pp. 761-779.

Kuhnimhof T., Zumkeller D., Chlond B. (2013), "Who Made Peak Car, and How? A Breakdown of Trends over Four Decades in Four Countries", Transport Reviews, vol. 33, n 3, pp. 325-342.

Kwan M. P., Kotsev A. (2015), "Gender Differences in Commute Time and Accessibility in Sofia, Bulgaria: A Study Using 3D Geovisualisation”, The Geographical Journal, vol. 181, $\mathrm{n}^{\mathrm{o}} 1$, pp. 83-96.

Maddala G. S. (1999), Limited-Dependent and Qualitative Variables in Econometrics, Cambridge, Cambridge University Press.

MARY-Portas F.-L. (2018). « Rhône et Métropole de Lyon : des territoires contrastés avec une bonne dynamique de l'emploi », Insee Flash Auvergne-Rhône-Alpes, n ${ }^{\circ} 34$. 
La mobilité domicile-travail des actifs de l'aire urbaine lyonnaise : une approche temporelle (1995-2015)

McGuckin N., Murakami E. (1999), "Examining Trip-Chaining Behavior: Comparison of Travel by Men and Women", Transportation Research Record, n ${ }^{\circ}$ 1693, pp. 79-85.

Metz D. (2013), "Peak Car and Beyond: The Fourth Era of Travel”, Transport Reviews, vol. 33, $\mathrm{n}^{\mathrm{o}} 3$, pp. 255-270.

MinNi C. (2012), « Les disparités sur le marché du travail entre les femmes et les hommes : une analyse sur longue période », Dares analyses, $n^{\circ} 015$.

OAKIl A. T. M., Manting D., NiJland H. (2016), "Determinants of Car Ownership among Young Households in the Netherlands: The Role of Urbanisation and Demographic and Economic Characteristics", Journal of Transport Geography, vol. 51, pp. 229-235.

OBSERVATOIRE DES TERRITOIRES (2016), Emploi et territoires. Rapport de l'Observatoire des territoires 2016, Paris, Commissariat général à l'égalité des territoires.

OBSERVATOIRE PARTENARIAL DÉPLACEMENTS (2012), « Les déplacements domicile-travail, une illustration du fonctionnement des territoires », Transports et mobilité, $\mathrm{n}^{\circ} 7$, Lyon, Agence d'urbanisme pour le développement de l'agglomération lyonnaise.

Olmo Sanchez I., Maeso Gonzalez E. (2016), "Gender Differences in Commuting Behavior: Women's Greater Sensitivity”, Transportation Research Procedia, vol. 18, pp. 66-72.

Ortar N., Boudreau J.-A., Vincent-Geslin S., Ribeiro C., Bonnel P., Morency C., Pochet P., Bouzouina L., Martin T., Verreault H., LiCAJ I. (2015), Évolutions des pratiques de mobilité et du rapport à l'automobile chez les jeunes. Une comparaison Lyon-Montréal, Rapport final de recherche, Laboratoire d'économie et d'aménagement des transports, Laboratoire Vespa, Polytechnique Montréal pour le compte du Forum vies mobiles.

Pasquier R., Guigner S., Cole A. (dir.) (2011), Dictionnaire des politiques territoriales, Paris, Presses de Sciences Po.

SARI F. (2011), « Expliquer les déplacements domicile-travail en Île-de-France : le rôle de la structure urbaine et des caractéristiques socio-économiques », Les Cahiers scientifiques du transport, $\mathrm{n}^{\circ} 60$, pp. 123-156.

Scheiner J., Holz-Rau C. (2012), "Gender Structures in Car Availability in Car Deficient Households", Research in Transportation Economics, vol. 34, n 1, pp. 16-26.

Vincent S., Licaj I., Pochet P., Bonnel P., Bouzouina L., Ortar N. (2014), Les Jeunes Adultes et la voiture. Analyse des enquêtes ménages déplacements de la région grenobloise de 2002 et 2010, Projet EvolMob, rapport d'étape pour le Forum vies mobiles, LET.

WAARD J. VAN DER, Jorritsma P., IMMERs B. (2013), "New Drivers in Mobility: What Moves the Dutch in 2012?", Transport Reviews, vol. 33, n 3, pp. 343-359. 


\section{ANNEXE}

TABLEAU - Résultats des modèles économétriques expliquant les pratiques de mobilité quotidienne, pour les actifs de l'agglomération lyonnaise

\begin{tabular}{|c|c|c|c|c|c|c|}
\hline & \multicolumn{3}{|c|}{ Distance domicile-travail } & \multicolumn{3}{|c|}{ Usage de la voiture } \\
\hline & \multirow{2}{*}{$\begin{array}{c}\begin{array}{c}\text { Effet } \\
\text { principal }\end{array} \\
(1995)\end{array}$} & \multicolumn{2}{|c|}{$\begin{array}{l}\text { Termes d'interaction } \\
\text { avec la vague }\end{array}$} & \multirow{2}{*}{\begin{tabular}{|c|}
$\begin{array}{c}\text { Effet } \\
\text { principal }\end{array}$ \\
$(1995)$
\end{tabular}} & \multicolumn{2}{|c|}{$\begin{array}{l}\text { Termes d'interaction } \\
\text { avec la vague }\end{array}$} \\
\hline & & 2006 & 2015 & & 2006 & 2015 \\
\hline Femme & $-1,067^{* * *}$ & 0,090 & 0,309 & $-0,735^{* * *}$ & 0,187 & $0,454^{* * * *}$ \\
\hline Âge & 0,018 & $0,140^{*}$ & 0,078 & $0,098^{* * *}$ & $-0,088^{*}$ & 0,003 \\
\hline$\hat{A}^{2} e^{2}$ & $-0,001$ & $-0,002^{*}$ & $-0,0007$ & $-0,001^{* * * *}$ & $0,001^{*}$ & 0,0004 \\
\hline \multicolumn{7}{|l|}{ Composition du ménage } \\
\hline Célibataire & Réf. & Réf. & Réf. & Réf. & Réf. & Réf. \\
\hline En couple sans enfant & 0,113 & $-0,360$ & $-0,093$ & $0,912^{* * * 4}$ & $-0,607^{* * 3}$ & $-0,592^{*}$ \\
\hline Famille monoparentale & 0,328 & $-0,816^{*}$ & 0,208 & $1,782^{* * *}$ & $-1,191^{* * *}$ & $-1,024^{* * * *}$ \\
\hline Famille 1 enfant, moins de 5 ans & 0,192 & $-0,142$ & $-0,767$ & $0,970^{\text {***4 }}$ & $-0,409$ & $-0,453$ \\
\hline Famille 1 enfant, de plus de 5 ans & 0,256 & $-0,152$ & 0,032 & $1,490^{* * *}$ & $-1,032^{* * *}$ & $-1,049^{* * * *}$ \\
\hline Famille 2 enfants, dont moins de 5 ans & 0,510 & $-0,547$ & 0,539 & $1,487^{* * *}$ & $-0,527$ & $-0,900^{* * * *}$ \\
\hline Famille 2 enfants, sans moins de 5 ans & 0,416 & $-0,554$ & 0,063 & $1,983^{* * * *}$ & $-1,440^{* * *}$ & $-1,563^{* * *}$ \\
\hline Famille 3 enfants ou plus, dont moins de 5 ans & $-0,304$ & $-0,059$ & $-0,374$ & $-0,752^{* * *}$ & $0,979^{\text {*an }}$ & $0,671^{*}$ \\
\hline Famille 3 enfants ou plus, sans moins de 5 ans & 0,234 & $-0,490$ & $-0,190$ & $2,255^{* *}$ & $-1,975^{* * *}$ & $-1,732^{* * * *}$ \\
\hline A un conjoint en emploi & 0,066 & 0,061 & 0,016 & $-0,304^{* *}$ & 0,199 & $0,335^{*}$ \\
\hline \multicolumn{7}{|l|}{ Profession et catégorie socioprofessionnelle } \\
\hline Entrepreneur, artisan, commerçant & $-1,946^{* * *}$ & 0,695 & 0,046 & $-0,100$ & $0,585^{*}$ & 0,143 \\
\hline Cadre et profession intellectuelle & 0,251 & 0,266 & 0,116 & 0,295 & $-0,218$ & $-0,794$ \\
\hline Profession intermédiaire & 0,174 & 0,324 & $-0,435$ & $0,455^{* * *}$ & $-0,203$ & $-0,821^{* * * *}$ \\
\hline Employé & $-0,076$ & $-0,196$ & $-0,285$ & $-0,209$ & $-0,026$ & $-0,547^{* * * *}$ \\
\hline Ouvrier & Réf. & Réf. & Réf. & Réf. & Réf. & Réf. \\
\hline \multicolumn{7}{|l|}{ Temps de travail } \\
\hline Temps plein & Réf. & Réf. & Réf. & Réf. & Réf. & Réf. \\
\hline Temps partiel & $-1,025^{* * *}$ & 0,090 & $-0,116$ & $-0,121$ & $-0,239$ & $-0,330$ \\
\hline \multicolumn{7}{|l|}{ Lieu de résidence } \\
\hline Centre-ville & $-2,652^{* * * *}$ & $-0,735^{* * *}$ & $-2,229^{* * * * *}$ & $-0,714^{* * *}$ & $-0,703^{* * *}$ & $-1,553^{* * * *}$ \\
\hline $1^{\text {re }}$ couronne & $-1,807^{* * *}$ & $-0,678^{* * *}$ & $-1,535^{* * *}$ & $-0,012$ & $-0,561^{* * *}$ & $-1,257^{* * *}$ \\
\hline $2^{e}$ couronne & Réf. & Réf. & Réf. & Réf. & Réf. & Réf. \\
\hline
\end{tabular}


La mobilité domicile-travail des actifs de l'aire urbaine lyonnaise : une approche temporelle

(1995-2015)

\begin{tabular}{lrrr|rrr}
\hline & \multicolumn{3}{c|}{ Distance domicile-travail } & \multicolumn{3}{c}{ Usage de la voiture } \\
\hline & $\begin{array}{c}\text { Effet } \\
\text { principal }\end{array}$ & $\begin{array}{c}\text { Termes d'interaction } \\
\text { avec la vague }\end{array}$ & $\begin{array}{c}\text { Effet } \\
\text { principal }\end{array}$ & $\begin{array}{c}\text { Termes d'interaction } \\
\text { avec la vague }\end{array}$ \\
\hline Habite chez ses parents & $\mathbf{( 1 9 9 5 )}$ & $\mathbf{2 0 0 6}$ & $\mathbf{2 0 1 5}$ & $\mathbf{( 1 9 9 5 )}$ & $\mathbf{2 0 0 6}$ & $\mathbf{2 0 1 5}$ \\
\hline Accessibilité à la voiture & 0,539 & $-0,341^{*}$ & $-0,264$ & $-0,721^{* * * *}$ & $-0,145$ & 0,325 \\
\hline Permis & $0,470^{* *}$ & 0,367 & $1,122^{* * * *}$ & $4,116^{* * * *}$ & $-1,031^{* * * *}$ & $-0,528^{* * *}$ \\
\hline Jour de la semaine & $0,876^{* * *}$ & 0,137 & $-0,313$ & & & \\
Lundi & & & & & & \\
Mardi & Réf. & Réf. & Réf. & Réf. & Réf. & Réf. \\
Mercredi & 0,313 & $-0,362$ & 0,232 & $-0,186$ & 0,212 & 0,296 \\
Jeudi & 0,160 & $-0,505^{*}$ & $-0,176$ & $-0,060$ & $-0,063$ & 0,134 \\
Vendredi & $-0,051$ & $-0,117$ & 0,458 & $-0,289^{* * *}$ & 0,282 & 0,273 \\
\hline Constante & $0,911^{* *}$ & $-0,943^{* * * *}$ & $-0,292$ & 0,004 & $-0,059$ & 0,310 \\
\hline Nombre d'observations & $4,944^{* * * *}-1,979$ & 0,638 & $-3,732^{* * * *}$ & $2,730^{* * * *}$ & 1,003 \\
\hline
\end{tabular}

Note : les chiffres reportés dans ce tableau correspondent aux coefficients estimés des trois spécifications économétriques présentées dans l'encadré 2 . * : significatif à un seuil de $10 \%$, ** : significatif à un seuil de $5 \%$ et *** significatif à un seuil de $1 \%$.

Lecture : comme les modèles estimés sont non linéaires, les valeurs des coefficients ne quantifient pas directement l'effet de la variable associée. On peut néanmoins interpréter le sens de la relation grâce à la significativité et au signe du coefficient. Par exemple, les travailleurs à temps partiel ont, toutes choses étant égales par ailleurs, des distances domicile-travail effectuées plus faibles que les travailleurs à temps plein. En revanche, il n'y a pas de différence significative dans l'usage de la voiture pour se rendre au travail entre salariés à temps plein et salariés à temps partiel, à autres caractéristiques équivalentes.

Champ : individus en emploi, âgés de 18 à 70 ans, résidant et travaillant dans le périmètre 1995 de l'EMD de Lyon.

Source : EMD de Lyon 1995, 2006, 2015 ; calculs des auteurs. 
